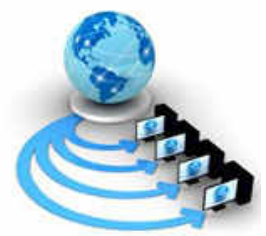

Volume 10, No. 1, January-February 2019

ISSN No. 0976-5697

International Journal of Advanced Research in Computer Science

RESEARCH PAPER

Available Online at www.ijarcs.info

\title{
DEMONSTRATING ELECTROCARDIOGRAM (ECG) SIGNAL PROCESSING TO SHOW NONLINEAR ABNORMALITIES OF THE HEART
}

\author{
Obinna, Eva N. \\ Dept. of Computer Science \\ Ignatius Ajuru University of Education Iwofe Rumuolumini \\ Obio Akpor, Nigeria
}

\author{
Kabari, Ledisi G. \\ Dept. of Computer Science \\ Ignatius Ajuru University of Education Iwofe Rumuolumini \\ Obio Akpor, Nigeria
}

\begin{abstract}
Heart beat classification is considered as the main tool for recognizing and diagnosing different heart diseases. The shape and size of these waves, the time between each wave and the rate and regularity of the heartbeat provide valuable information to doctors. An electrocardiogram, also called an ECG or EKG, is a quick, painless test that measures the heart's electrical activity and records any disturbances in heart rhythm. The paper aimed at demonstrating electrocardiogram (ECG) signal processing to show the nonlinear abnormalities of the heart using Microsoft Excel. The study will definitely enhance fast and cost effective diagnosis of Ischemic Episodes.
\end{abstract}

Keywords: Electrocardiogram, Arrhythmia, Heart Rhythm, Heart Anatomy, Cardiac Disorder.

\section{INTRODUCTION}

The human heart is an organ that pumps blood throughout the body using the circulatory system, removing carbon dioxide and wastes, and giving oxygen and nutrients to the tissues [1]. An abnormality of the heart is when the human heart beats too fast, slow, or irregularly [2]. It is called arrhythmia. The heart contains a complex system of values, nodes, and chambers that controls when and how the blood pumps [2].

There are different types of abnormal heart beat; they are: Atrial Fibrillation and Flutter, Congestive Heart Failure (CHF), Congestive (Dilated) Cardiomyopathy, Mitral Value Prolapse, Hypertensive Heart Disease, Cardiogenic Shock, Dissection of the Aorta, Hypokalemia, Hyperthyroidism, Anaphylaxis, Hypoglycemia (low blood sugar), Hypothyroidism, Aortic Coarctation, Ventricular Septal Defects [2]. The abnormal heart beat has various types. These ones mentioned are just few of it [2].

The abnormal heart beat considered here is the Ischemic Episodes. The word ischemia means heart stroke. It is a cardiovascular disorder which affects the heart and the blood vessels. The coronary arteries become narrowed by atherosclerosis which restricts the flow of blood and oxygen to the heart and brain. This makes brain cells to die which creates cardiac disorder or cardiac arrhythmias known as ischemia [3]. In detecting this heart disorder, the process takes longer if analyzed by the doctor using long duration ECG data. The key to ischemic episodes detection is the ST-segment deviation and T-wave Amplitude changes [4].

This work aimed at demonstrating electrocardiogram (ECG) signal processing to show the nonlinear abnormalities of the heart using Microsoft Excel.

\section{A. Medical Background of the Heart}

The heart consists of four chambers, two upper (the atria) and two lower (the ventricles) as shown in figure 1 . The heart is a powerful muscle that lies in the chest. The heart beat is the physical contraction of the heart muscle for pumping blood [5].
Abnormal heart rhythms is when an individual has an abnormal heart rhythm. It happens when an individual's heart electrical system breaks down or malfunctions. This may be a symptom of underlying coronary heart disease or other medical problems [6]. The causes of arrhythmias are: irritable heart cells, blocked signals, abnormal pathway, medicines and stimulants, coronary artery spasm (prinzmetalangina). Diagnosis of arrhythmias is done using electrocardiography (ECG) and Tilt Tests [6]. ECG shows the doctor how the electrical system in the heart works. Tilt tests helps the doctor to know whether or not different body positions will trigger an

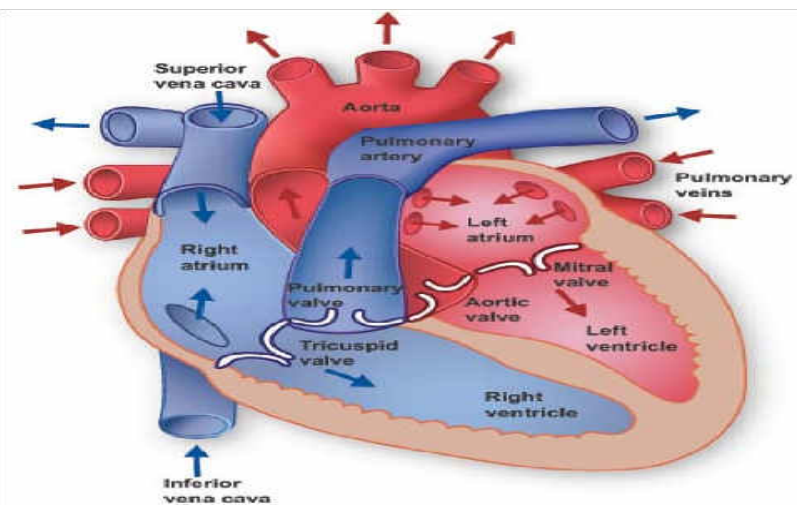

arrhythmia. They are useful for investigating the hearts of people who faint without explanation [6].

Figure 1. Hart Anatomy [7]

\section{B. Overview of an Abnormal Heart}

The heart is a muscular organ with four chambers designed to work efficiently, reliably, and continuously over a lifetime. The muscular walls of each chamber contract in a regulated sequence, pumping blood as required by the body while expending as little energy as possible during each heartbeat [8]. 
Heart disorders are the most common cause of an abnormal heart rhythm. Sometimes people are aware of abnormal heart rhythms, but many times they feel only their consequences, such as weakness or fainting [8]. The diagnosis is based on electrocardiography. Treatment involves restoring the heart to a normal rhythm and preventing further episodes.

Contraction of the muscle fibers in the heart is controlled by electricity that flows through the heart in a precise manner along distinct pathways at a controlled speed [8]. The electrical current that begins each heartbeat originates in the heart's pacemaker (called the sinus node or sino atrial node), located in the top of the upper right heart chamber (right atrium). The rate at which the pacemaker discharges the electrical current determines the heart rate. This rate is influenced by nerve impulses and by levels of certain hormones in the bloodstream.

\section{Conduction Systems}

The heart rate is regulated automatically by the autonomic nervous system, which consists of the sympathetic and parasympathetic divisions [8]. The sympathetic division increases the heart rate through a network of nerves called the sympathetic plexus. The parasympathetic division decreases the heart rate through a single nerve, the vagus nerve.

Heart rate is also influenced by hormones released into the bloodstream by the sympathetic division which is called epinephrine (adrenaline) and norepinephrine (noradrenaline). This sympathetic division's increases heart rate. Thyroid hormone also increases heart rate when it is been released into the blood stream by the thyroid gland [8].

When an adult is at rest, the normal heart rate is usually between 60 and 100 beats per minute. Lower rates may be normal in young adults, especially those who are physically fit. An individual's heart rate varies normally in response to exercise and such stimuli as pain and anger. Heart rhythm is considered abnormal only when the heart rate is inappropriately fast (called tachycardia), slow (called bradycardia), or irregular or when electrical impulses travel along abnormal pathways [8].

\section{Tracing the Heart's Electrical Pathway}

The sinoatrial (sinus) node (1) initiates an electrical impulse that flows through the right and left atria (2), making them contract. When the electrical impulse reaches the atrioventricular node (3), it is delayed slightly. The impulse then travels down the bundle of His (4), which divides into the right bundle branch for the right ventricle (5) and the left bundle branch for the left ventricle (5). The impulse then spreads through the ventricles, making them contract [8].



Figure 2. Tracing the Heart's Electrical Pathway [8]

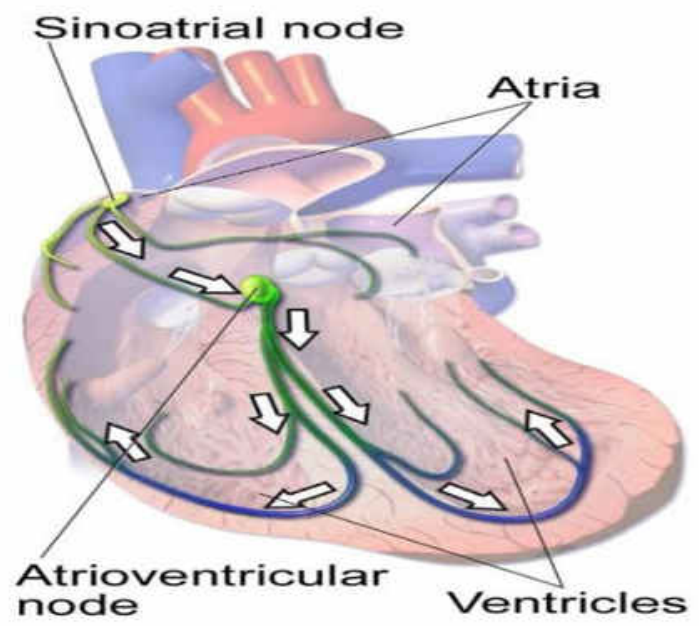

Figure 3. Electrical Conduction System of the Heart [8]

\section{E. Electrical Conduction System of the Heart}

The electrical current travels down the bundle of His which is a group of fibers that divide into a left bundle branch for the left ventricle and a right bundle branch for the right ventricle, after passing through the atrioventricular node. The electrical current then spreads in a regulated manner over the surface of the ventricles, from the bottom up, initiating contraction of the ventricles, which eject blood from the heart [8].

\section{F. Electro Cardiogram (ECG)}

This is the graphical recording of the electrical signals generated by the heart [9]. ECG was introduced for the first time by Willem Einthoven in 1983 The ECG mainly contains three main signal structure. Cardiac arrhythmias which are results of abnormal heart activity upon certain conditions can be indicated by the change in shape of one of these traces [6]. Placing electrodes (up to 12 electrodes) at various body points is the way in which the ECG can record the electrical activity of the heart [6]. An automation of cardiac arrhythmias using ECG is considered as one of the most important recent fields of research [10] as ECG is considered a reliable tool.

\section{DEMONSTRATION OF ECG SIGNAL PROCESSING TO SHOW NON LINEAR ABNORMALITIES OF THE HEART}

In order to demonstrate ECG signal processing to show nonlinear abnormalities of the heart, an abnormal heart data set was extracted online from kaggle database. This data contains various fields which includes; age, sex, cp, restbp, chol, fbs, restecg, thalach, exang, oldpeak, slope, and ca. This fields are described further in 2.1.

\section{A. Description of Fields Used in the Abnormal Heart Dataset}

The Age is in years, Sex ( $1=$ male; $0=$ female $)$, cp means chest pain type, restbp means resting blood pressure (in $\mathrm{mm}$ $\mathrm{Hg}$ on admission to the hospital), chol means serum cholestoral in $\mathrm{mg} / \mathrm{dl}$, fbs means ((fasting blood sugar $>120$ $\mathrm{mg} / \mathrm{dl}), \quad 1=$ true; $0=$ false), restecg means resting electrocardiographic results), thalach means ((maximum heart rate achieved), thal $3=$ normal; $6=$ fixed defect; $7=$ reversible defect, target 1 or 0 .), exang means (exercise induced angina 
$(1=$ yes; $0=$ no $)$, oldpeak means ST depression induced by exercise relative to rest, slope means the slope of the peak exercise ST segment, ca means number of major vessels (0-3) coloured by flourosopy. 2.2 shows the abnormal heart dataset.

\section{B. The Abnormal Heart Dataset}

Table 1 contains dataset of the abnormal heart. This abnormal heart dataset was extracted as a .csv file (heart.csv), which is a file extension name of Microsoft Excel.

Table I. Abnormal Heart Dataset [11]

\begin{tabular}{|c|c|c|c|c|c|c|c|c|c|c|c|c|}
\hline$A B$ & Sex & Chestpain & Restip cho & Fbs & & estecG :Ma & ExtR Exang & & Apeak Slope & $\mathrm{Ca}$ & Thal & AHD \\
\hline 1 & 63 & 1 typical & 145 & 2233 & 1 & 2 & 150 & 0 & 23 & 3 & 0 fixed & No \\
\hline 2 & 67 & 1 asymptomatic & 160 & 286 & 0 & 2. & 108 & 1 & 1.5 & 2 & 3 normal & Yes \\
\hline 3 & 67 & 1 asymptomatic & 120 & 23 & 0 & 2 & 129 & 1 & 26 & 2 & 2 reversable & Yes \\
\hline 4 & 37 & 1 nonanginal & 130 & 250 & 0 & 0 & 187 & 0 & 3.5 & 3 & Onormal & No \\
\hline 5 & 41 & a nontypical & 130 & 204 & 0 & 2) & 172 & 0 & 14 & 1 & Onormal & No \\
\hline 6 & 56 & 1 nontypical & 120 & 236 & 0 & 0 & 178 & 0 & 0.8 & 1 & Onormal & No \\
\hline$?$ & 62 & Q asymptomatic & 190 & 268 & 0 & 2 & 160 & 0 & 3.6 & 3 & 2 normal & Yes \\
\hline 8 & 37 & 00 asymptomatic & 120 & 354 & 0 & 0 & 163 & 1 & 0.6 & 1 & O normal & No \\
\hline 9 & 63 & I asymptomatic & 130 & 254 & 0 & 2 & 147 & 0 & 14 & 2 & 1 reversable & res \\
\hline 10 & 53 & 1 asymptomatic & 140 & 203 & 1 & 2 & 155 & 1 & 3.1 & 3 & Oreversable & Yes \\
\hline 11 & 57 & 1. asymptomatic & 140 & 192 & a & 0 & 148 & 0 & 0.4 & 2 & Of fixed & № \\
\hline 12 & 36 & Onontypical & 100 & 294 & 0 & 2 & 153 & 0 & 1.3 & 2 & Onormal & No \\
\hline 13 & 56 & I nonanglnal & 130 & 256 & 1 & 2 & 142 & 1 & 0.6 & 2 & 1 fixed & Yes \\
\hline 14 & 44 & 1 nontypical & 120 & 263 & 0 & 0 & 173 & 0 & 0 & 1 & O reversable & No \\
\hline 15 & 52 & 1 nonanginal & 172 & 199 & 1 & 0 & 162 & 0 & 0.5 & 1 & Oreverssble & No \\
\hline 16 & 57 & 1 norangenal & 150 & 168 & 0 & 0 & 174 & 0 & 1.5 & 1 & 0 normal & No \\
\hline 1) & 48 & 1 nontypical & 110 & 29 & 0 & 0 & 168 & 0 & 1 & 3 & 0 reversable & Yes \\
\hline 18 & 54 & 1 asymptomatic & 140 & 239 & 0 & $\theta$ & 150 & 0 & 1.2 & 1 & Onormal & No \\
\hline 19 & 48 & a nonanginal & 130 & 275 & 0 & 0 & 139 & 0 & 0.2 & 1 & O formal & No \\
\hline$x$ & 49 & 1 nontypical & 130 & 266 & 0 & 0 & 171 & 0 & 0.6 & 1 & On normal & No \\
\hline a1 & 64 & 1 typical & 110 & 211 & 0 & 2 & 144. & 1 & 1.8 & 2 & Onomal & No \\
\hline 22 & 58 & Otypial & 150 & 283 & 1 & 2 & 152 & 0 & 1 & 1 & Onomal & No \\
\hline 23 & 58 & I nontypical & 120 & 204 & 0 & 2 & 150 & 0 & 1.6 & 2 & Onormal & Yes \\
\hline 24 & 58 & 1 nonanginal & 132 & 224 & 0 & 2 & 173 & 0 & 3.2 & 1 & 2 reverssole: & Yes \\
\hline 25 & 60 & 1 asymptomatic & 130 & 206 & 0 & 2 & 132 & 1 & 2.4 & 2 & 2 reversable & Yes \\
\hline 20 & 50 & 0 nonangenal & 120 & 213 & 0 & 0 & 158 & 0 & 1.6 & 2 & 0 nornal & No \\
\hline 27 & 58 & O nonanginal & 120 & 340 & 0 & a & $1 \pi$ & 0 & 0 & 1 & 0 normal & No \\
\hline 28 & 66 & otyplat & 150 & 226 & 0 & 0 & 114 & 0 & 26 & 3 & Onomal & No \\
\hline 29 & 43 & 1 asymptomatic & 150 & 247 & 0 & 0 & 171 & 0 & 1.5 & 1 & Inormal & No \\
\hline 30 & 40 & 1 asymptomatic & 110 & 167 & 0 & 2 & 114 & 1 & 2 & 2 & Oreversable & Yes \\
\hline $3 !$ & 69 & Otypical & 140 & 239 & 0 & 0 & 151 & 0 & 18 & 1 & Enormal & No \\
\hline 32 & 60 & 1.:symptomatic & 117 & 230 & 1 & 0 & 160 & 1 & 1.4 & 1 & 2 reversable & Yes \\
\hline 33 & 64 & 1 nonangeinal & 140 & 335 & 0 & 0 & 158 & 0 & 0 & 1 & Onormal & Yes \\
\hline 34 & 59 & 1 ssymptomatic & 135 & 234 & 0 & 0 & 161 & 0 & 0.5 & 2 & O reversable & $\mathrm{No}$ \\
\hline 35 & 44 & 1 nonarginal & 130 & 233 & 0 & 0 & 179 & 1 & 0.4 & 1 & Anormal & No \\
\hline 36 & 42 & 1 asymptomatic & 140 & 226 & 0 & 0 & 178 & 0 & 0 & 1 & Onormal & No \\
\hline 37 & 43 & 1 asymptomatic & 120 & 177 & 0 & 2 & 120 & 1 & 25 & 2 & o reverastle & Yes \\
\hline 38 & 57 & 1 asymptomatic & 150 & 276 & 0 & 2 & 112 & 1 & 0.6 & 2 & If fixed & Yes \\
\hline 39 & 55 & 1 asymptomatic & 132 & 353 & 0 & 0 & 132 & 1 & 1.2 & 2 & I revertsale & Yes \\
\hline 40 & 61 & 1 nonanginal & 150 & 243 & 1 & e & 137 & 1 & 1 & 2 & Onormal & No \\
\hline 41 & 65 & 0.85ymptomatic & 150 & 225 & 0 & l & 114 & 0 & 1 & 2 & 3 reversable & Yes \\
\hline 42 & 40 & 1 typical & 140 & 199 & 0 & 0 & 178 & 1 & 1.4 & 1 & O reversable & No \\
\hline 43 & 71 & O nontypleal & 160 & 302 & 0 & 0 & 162 & 0 & 0.4 & 1 & 2normal & No \\
\hline 44 & 59 & 1 nonarginal & 150 & 212 & 1 & 0 & 157 & 0 & 16 & 1 & Onormal & No \\
\hline 45 & 62 & 0 asymiptonatic & 130 & 330 & 0 & 2 & 169 & 0 & 0 & 1 & Onormal & Yes \\
\hline 45 & 58 & 1 nonarginal & 112 & 230 & 0 & 2 & 165 & 0 & 2.5 & 2 & 1 reversable & Yes \\
\hline 47 & 51 & 1 nonarginal & 110 & 175 & 0 & 0 & 123 & 0 & 0.6 & 1 & Onormal & No \\
\hline 48 & 50 & 1 aspmptomatic & 150 & 243 & 0 & 2 & 128 & 0 & 26 & 2 & Oreversable & Yes \\
\hline 49 & 65 & On nobaraginal & 140 & 417 & 1 & 2 & 157 & 0 & 0.8 & 1 & Inormal & No \\
\hline 50 & 53 & 1 nonarginal & 130 & 197 & 1 & 2 & 152 & 0 & 1.2 & 3 & Onormal & No \\
\hline 51 & 41 & Onofitypical & 105 & 198 & 0 & 0 & 168 & 0 & 0 & 1 & I normal & No \\
\hline 52 & 65 & 1 asymptomatic & 120 & 177 & 0 & 0 & 140 & 0 & 0.4 & 1 & Oreverssble & No \\
\hline 53 & 4 & 1 asymptomatic & 112 & 290 & 0 & 2 & 153 & 0 & 0 & 1 & I normal & Yes \\
\hline 54 & $\mu$ & 1 nontypical & 130 & 219 & 0 & 2 & 188 & 0 & 0 & 1 & Onormal & No \\
\hline 55 & 60 & 1. symmotomatic & 130 & 253 & 0 & 0 & 144 & 1 & 1.4 & 1 & I reversable & Yes \\
\hline
\end{tabular}
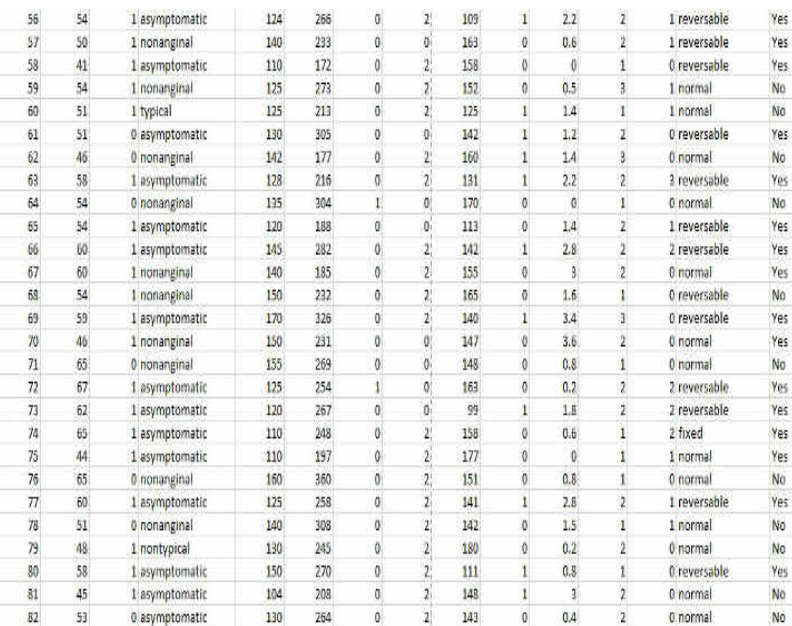
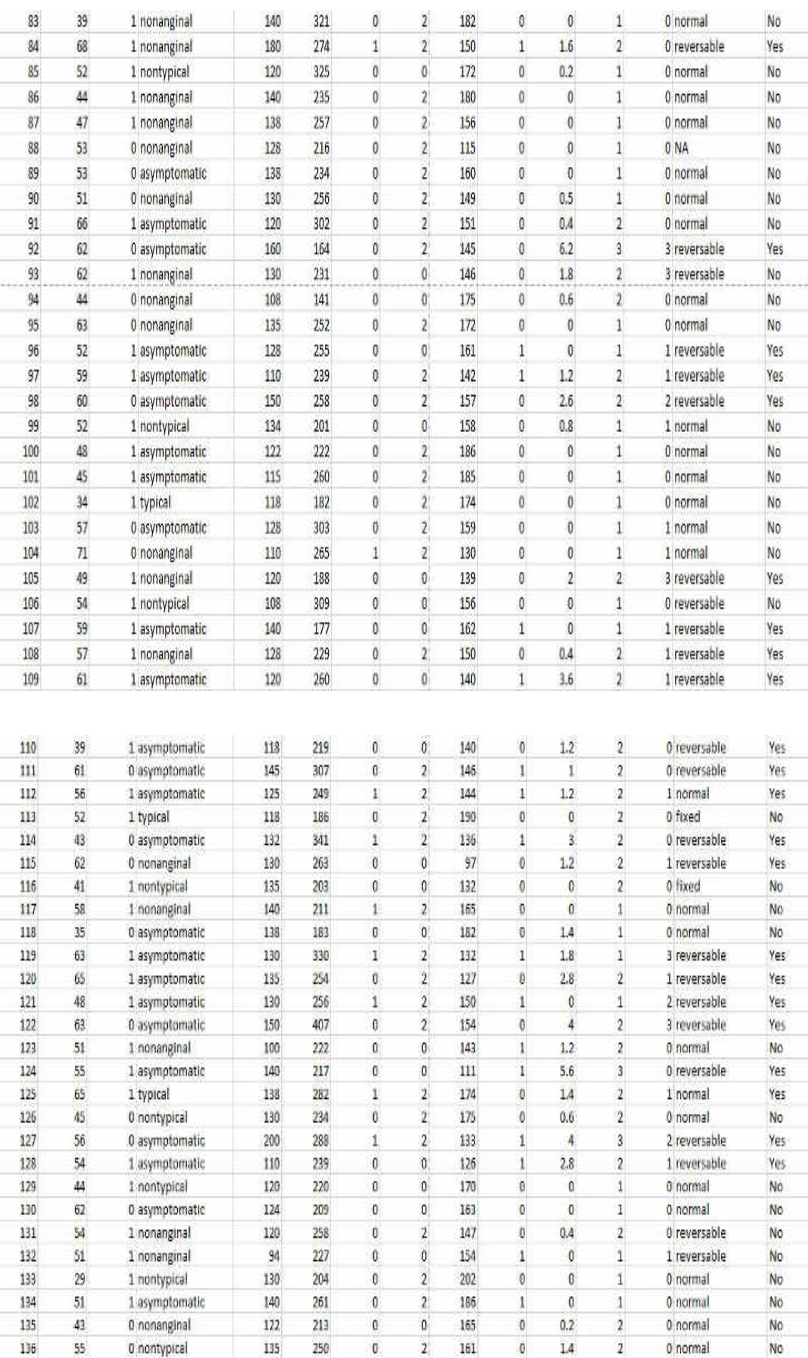
Obinna, Eva N. et al, International Journal of Advanced Research in Computer Science, 10 (1), Jan-Feb 2019,43-49
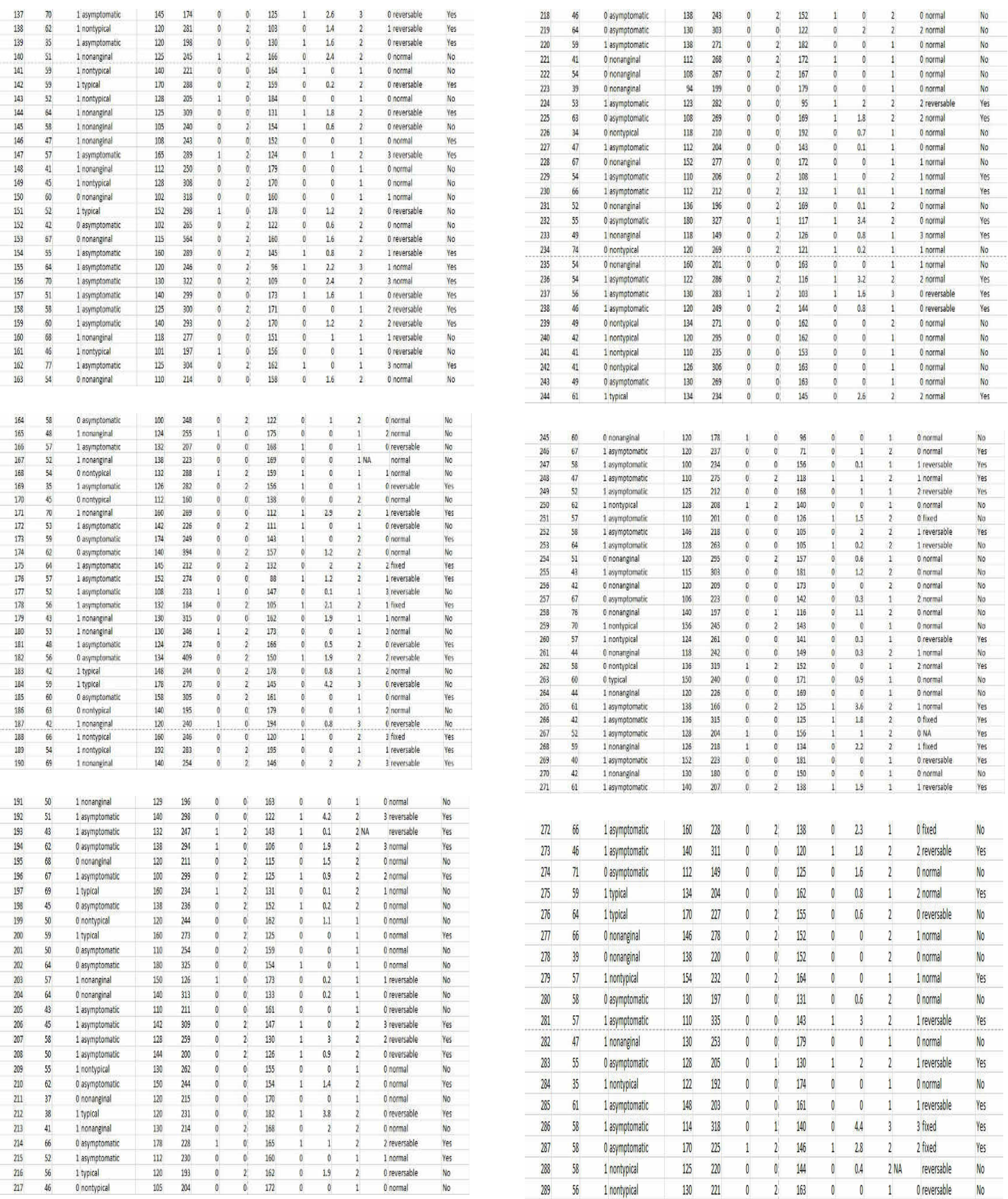

\begin{tabular}{|c|c|c|c|c|c|c|c|c|c|c|c|c|}
\hline 22 & 66 & 1 asmmptomatic & 160 & 228 & 0 & 2 & 138 & 0 & 2.3 & 1 & Ofixed & No \\
\hline 273 & 46 & 1 asymptomatic & 140 & 311 & 0 & 0 & 120 & 1 & 1.8 & 2 & 2 reverasable & Yes \\
\hline 274 & 71 & Dasmmitonatic & 112 & 149 & 0 & 0 & 125 & 0 & 1.6 & 2 & Onombl & No \\
\hline 275 & 59 & 1 thpical & 134 & 204 & 0 & 0 & 162 & 0 & 0.8 & 1 & 2 nommal & Yes \\
\hline 276 & 64 & 1tipical & 170 & 277 & 0 & 2 & 155 & 0 & 0.6 & 2 & Oreversable & No \\
\hline 277 & 66 & Onomargingal & 145 & 288 & 0 & 2 & 152 & 0 & 0 & 2 & Inomal & No \\
\hline 278 & 39 & Onomanginal & 138 & 200 & 0 & 0 & 152 & 0 & 0 & 2 & Onombl & No \\
\hline 279 & 57 & 1 1 nompipical & 154 & 232 & 0 & 2 & 164 & 0 & 0 & 1 & 1 nommal & Yes \\
\hline 280 & 58 & Oasmmptonatic & 130 & 197 & 0 & 0 & 131 & 0 & 0.6 & 2 & Onomel & No \\
\hline 281 & 57 & 1 asmmotonatic & 110 & 335 & 0 & 0 & 143 & 1 & 3 & 2 & 1.rversable & Yes \\
\hline 282 & 47 & In nonarginal & 130 & 253 & 0 & 0 & 179 & 0 & 0 & 1 & Onommal & No \\
\hline 283 & 55 & Oasmmptomatic & 128 & 205 & 0 & 1 & 130 & 1 & 2 & 2 & 1 reverasalle & Yes \\
\hline 284 & 35 & Inontypical & 122 & 192 & 0 & 0 & 174 & 0 & 0 & 1 & Onomal & No \\
\hline 285 & 61 & 1 asmmptonatic & 148 & 203 & 0 & 0 & 161 & 0 & 0 & 1 & Ireverable & yes \\
\hline 286 & 58 & 1 asmmotonatic & 114 & 318 & 0 & 1 & 140 & 0 & 4.4 & 3 & 3 fixed & Yes \\
\hline 287 & 58 & Oasymptonatic & 170 & 225 & 1 & 2 & 146 & 1 & 2.8 & 2 & 2fiked & Yes \\
\hline 288 & 58 & 1 nontypical & 125 & 200 & 0 & 0 & 14 & 0 & 0.4 & $2 \mathrm{Na}$ & reversable & No \\
\hline 289 & 56 & 1 nonippical & 130 & 211 & 0 & 2 & 163 & 0 & 0 & 1 & Oreverabale & No \\
\hline
\end{tabular}




\begin{tabular}{|c|c|c|c|c|c|c|c|c|c|c|c|c|}
\hline 290 & 56 & 1 nontypical & 120 & 240 & 0 & 0 & 169 & 0 & 0 & 3 & Onormal & No \\
\hline 291 & 67 & 1 nonargenal & 152 & 212 & 0 & 2 & 150 & 0 & 0.8 & 2 & Oreversable & Yes \\
\hline 292 & 55 & O nontypical & 132 & 342 & 0 & 0 & 166 & 0 & 1.2 & 1 & Onormal & No \\
\hline 293 & 4 & 1 asymptomatic & 120 & 169 & 0 & 0 & 144 & 1 & 28 & 3 & of fixed & Yes \\
\hline 294 & 63 & 1 asymptomatic & 140 & 187 & 0 & 2 & 14 & 1 & 4 & 1 & 2reversable & Yes \\
\hline 205 & 63 & 0 asymptomantic & 124 & 197 & 0 & 0 & 136 & 1 & 0 & 2 & Onomial & Yes \\
\hline 296 & 41 & 1 nontypical & 120 & 157 & 0 & 0 & 182 & 0 & 0 & 1 & O normal & No \\
\hline 297 & 59 & 1 asymptomatic & 164 & 176 & 1 & 2 & 90 & 0 & 1 & 2 & 2 fixed & Yes \\
\hline 288 & 57 & 0 asymptomatic & 140 & 241 & 0 & 0 & 123 & 1 & 0.2 & 2 & D reversable & Yes \\
\hline 299 & 45 & 1tppical & 110 & 264 & 0 & 0 & 132 & 0 & 1.2 & 2 & D reversable & Yes \\
\hline 300 & 68 & 1 asymptomatic & 14 & 198 & 1 & 0 & 141 & 0 & 3.4 & 2 & 2 reversable & Yes \\
\hline 301 & 57 & 1 asymptomatic & 130 & 131 & 0 & 0 & 115 & 1 & 1.2 & 2 & 1 reversisble & Yes \\
\hline 302 & 57 & O nontypical & 130 & 236 & 0 & 2 & 174 & 0 & 0 & 2 & 1 normal & Yes \\
\hline 303 & 38 & 1 nonarginal & 138 & 175 & 0 & 0 & 173 & 0 & 0 & $1 \mathrm{NA}$ & normal & No \\
\hline
\end{tabular}

\section{Demonstration of Abnormal Heart Dataset Using Excel}

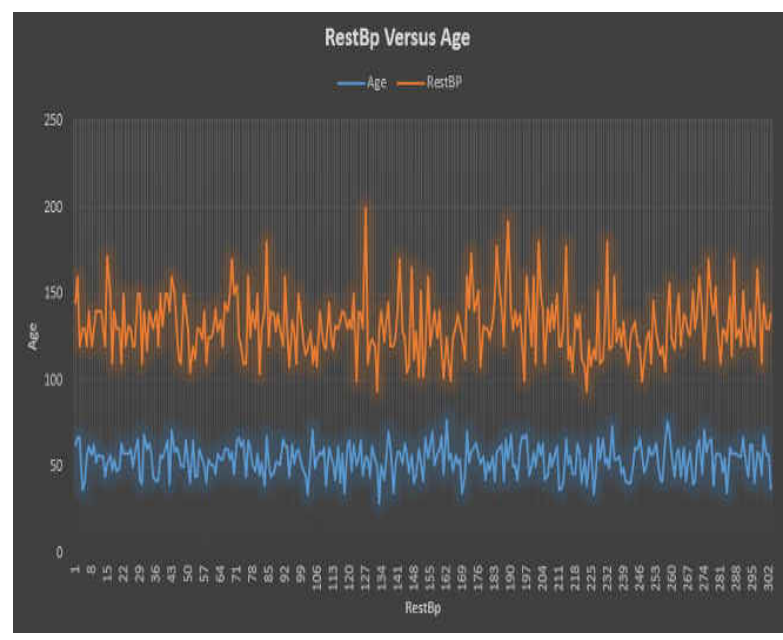

Figure 4. Demonstrating RestBp versus Age

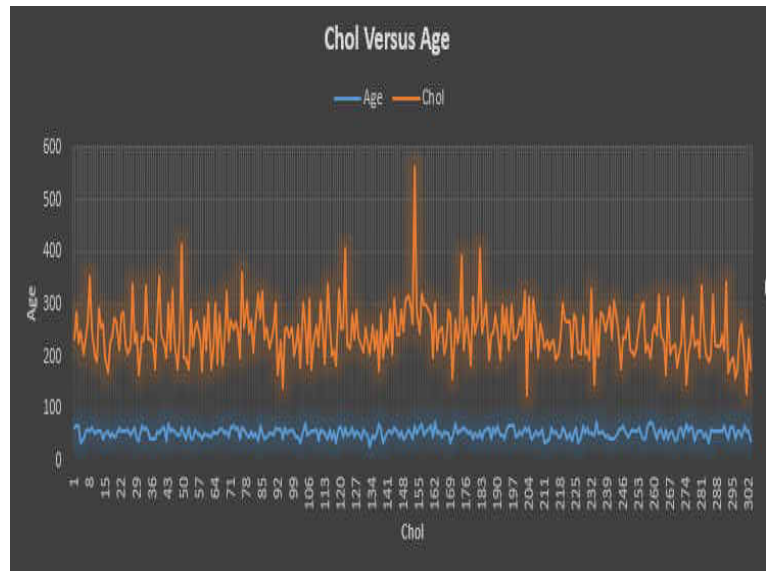

Figure 5. Demonstrating Chol versus Age

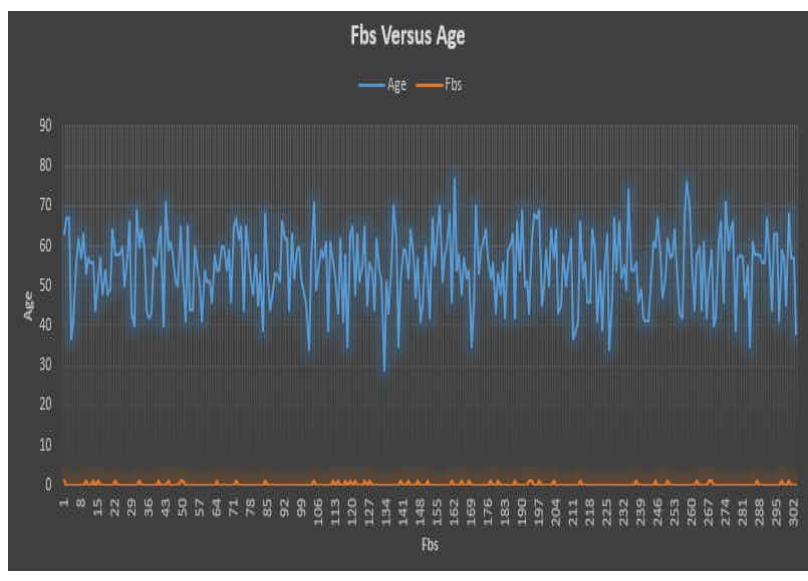

Figure 6. Demonstrating Fbs versus Age

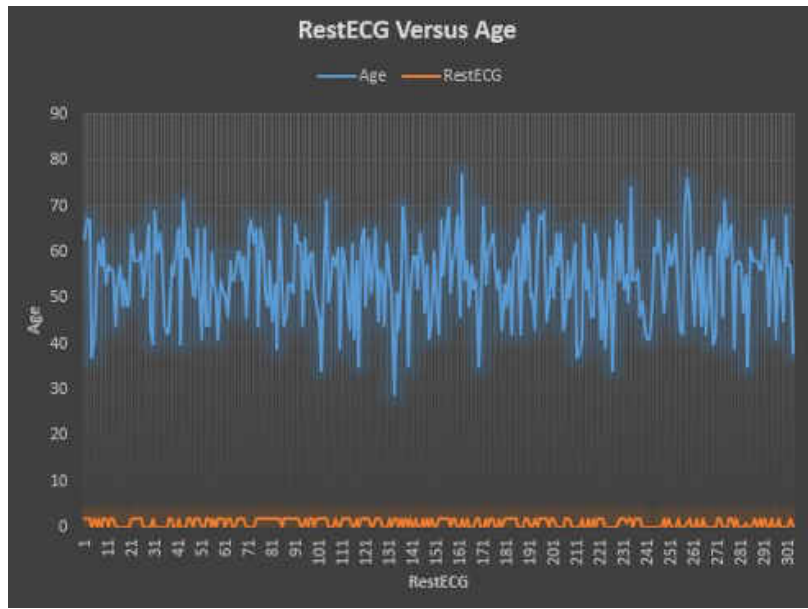

Figure 7. Demonstrating RestECG versus Age

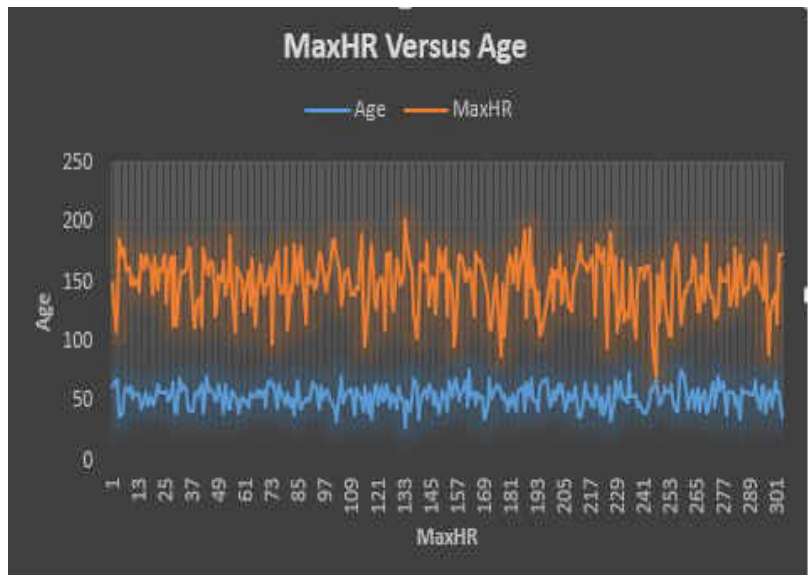

Figure 8. Demonstrating MaxHR versus Age 


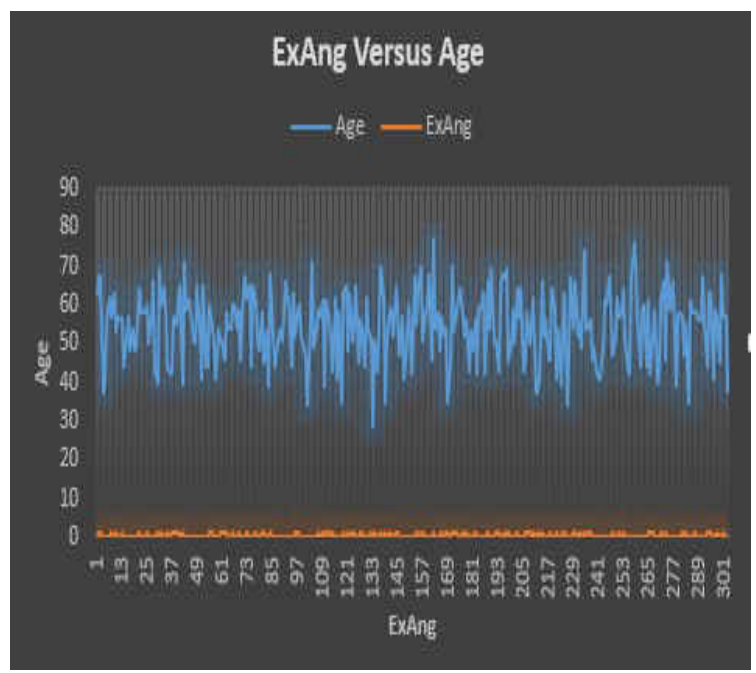

Figure 9. Demonstrating ExAng versus Age

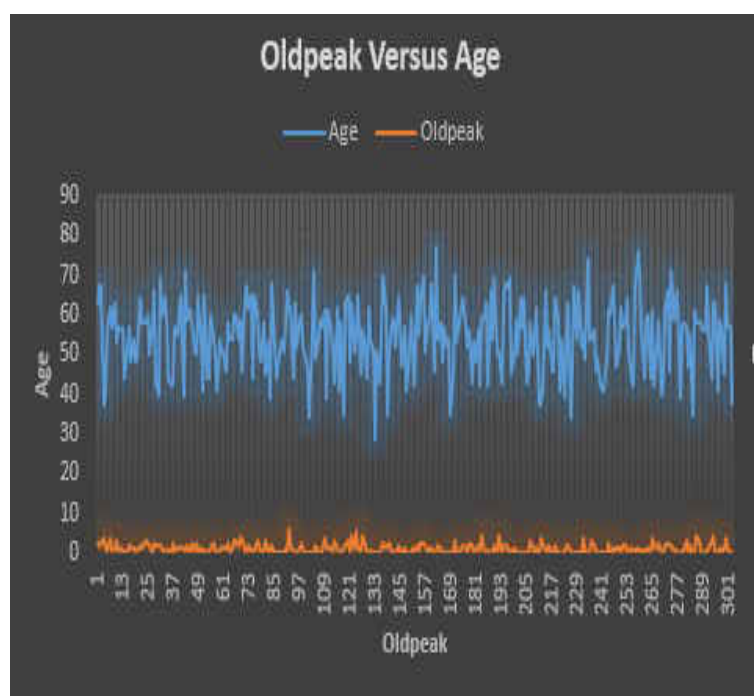

Figure 10. Demonstrating Oldpeak versus Age

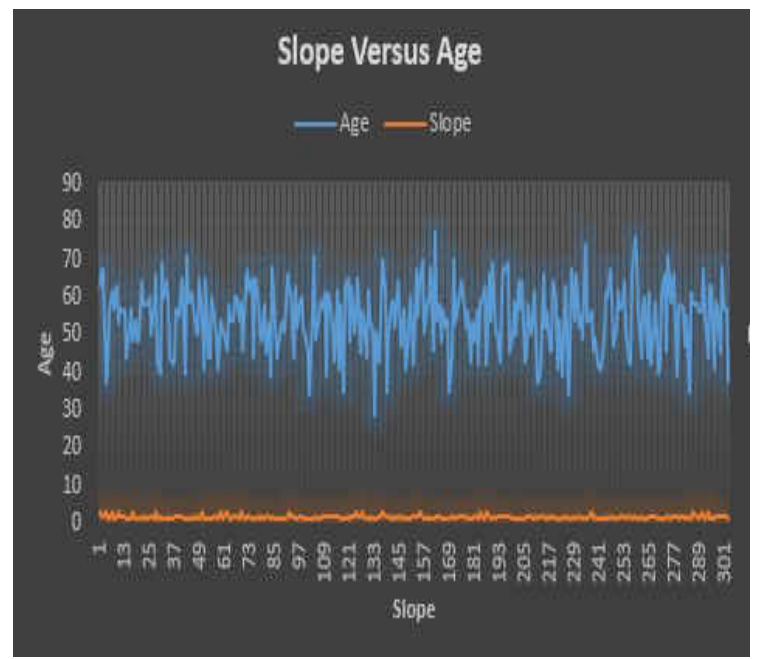

Figure 11. Demonstrating Slope versus Age

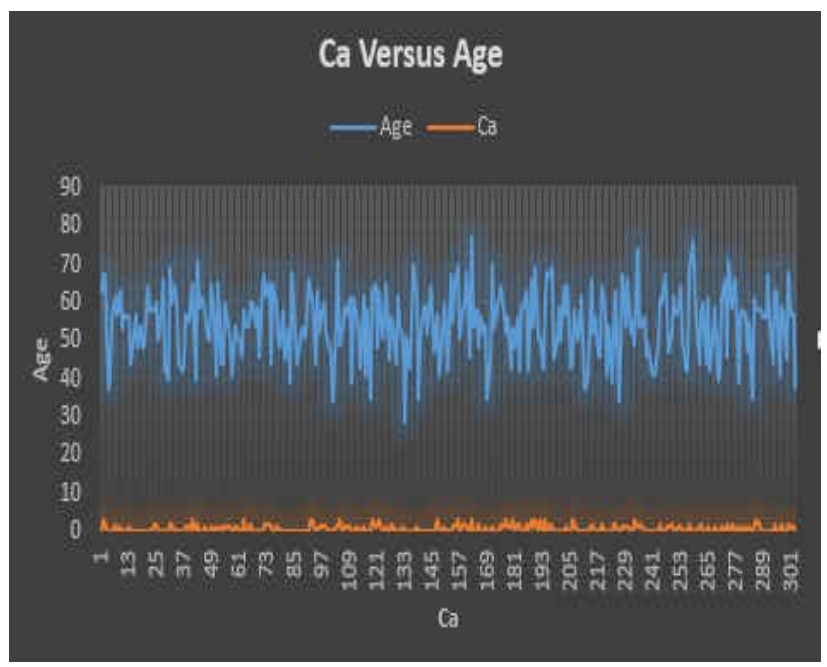

Figure 12. Demonstrating Ca versus Age

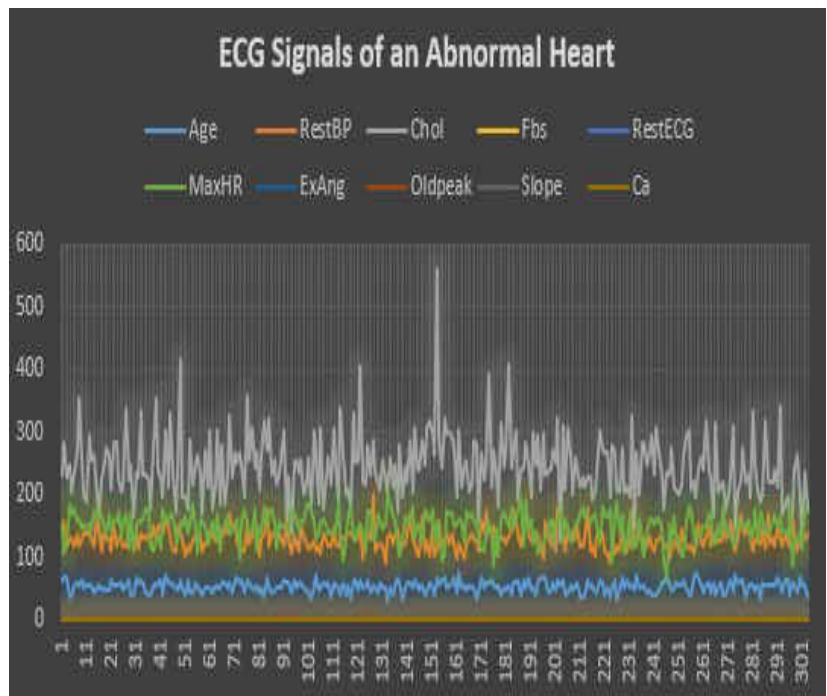

Figure 13. ECG Signals of an Abnormal Heart

\section{CONCLUSION}

The heart is an important and sensitive organ of the human body and should be prevented from any form of hazardous agent. Every individual should do a routine check of their body system so as to know the health status of their body. That way they can also be able to know the condition of their heart from time to time. The ECG is an important signal among all bioelectrical signals used in the diagnosis of many cardiac disorders and can be recorded from the wave passage of the depolarization and repolarization processes in the heart. The voltage in the heart tissues is conducted to the body surface where it is measured using electrodes. This work demonstrated an ECG Signal Processing of an abnormal heart Using Microsoft Excel. In future this demonstration can be done using Mat Lab or Python programming language. Analysis can also be conducted using any of the machine language model.

\section{REFERENCES}

[1] T. Lewis," Human Heart: Anatomy, Function and Facts." Staff writer Live Science 2016. 
[2] S. Debra, "What causes Abnormal Heart Rhythms." Health line. 2017

[3] T. Rocha, S. Paredes, P. Carvalho, J. Henriques, M. Harris, J. Morais, M. Antumes, "A lead dependent ischemic episodes detection strategy using Hermite functions. "Biomedical Signal Processing and control, 5(4), pp. 271-281, 2010.

[4] C. Papaloukas, D. I. Fotiadis, A. Likas, L. K. Michalis, "Automated Detection of Ishemia Detection in Long Duration from ECGs.” Journal on Cardiovasc Rev Rep, 24(6), pp. 313-320. 2003.

[5] M. K. Tantawi, K. Revett, A. B. Salem, M. F. Tolba, "Electrocardiogram (ECG): A New Burgeoning Utility for Biometric Recognition." In: Bio- inspiring Cyber Security and Cloud Services; Trends and Innovations, A.E. Hassanien, K. T. Hoon, K. Janusz, A. A.Ismail, (Eds). Springer, Berlin, Germany, ISBN: 978-3-66243615-8, pp: 349-382. 2014.

[6] Arrhythmias (abnormal heart rhythms). Retrieved from healthywa.wa.gov.au/Articles /AE/Arrhythmias- abnormal - heart-rhythms on 16/10/2018.

[7] H. El-Saadawy, M. Tantawi, A. H. Shedeed, F. M. Tolba, "Heart Disease Diagnosis using Electrocardiogram (ECG)
Signal Processing. Asian Journal of Information Technology. 16(10): 771-782, ISSBN: 1682-3915 Ain Shams University, Cairo, Egypt. 2017.

[8] M. L. Brent, "Overview of Abnormal Heart Rhythms." MD, Professor of Medicine, Department of Cardiac Services, Libin Cardiovascular Institute of Alberta, University of Calgary. Merck Sharp \& Dohme Corp. a Subsidiary of Merck \& Co. inc. Kenilworth, $\mathrm{Nj}$. USA. 2018.

[9] R. U. Acharya, J. Suri, J. A. E. Span, “Advances in Cardiac Signal Processing.” Springer Verlag. 2007

[10] H. Yazdanian, A. Normani, M. R. Yazdchi, "Autonomous detection of heartbeats and categorizing them by using Support Vector Machines." Proceedings of the 20th Iranian Conference on Biomedical Engineering (ICBME). IEEE, Isfahan, Iran, ISBN: 978-1-4799-3233-7, pp: 223-227. 2013.

[11] API Kaggle datasets download -d Zhaoyingzhu/heart.csv retrieved from https:/github.com/kaggle/kaggle-api on $16 / 10 / 2018$. 